\title{
Motivation and detectability of an invisibly decaying Higgs boson at the Fermilab Tevatron
}

\author{
Stephen P. Martin \\ Department of Physics, Northern Illinois University, DeKalb, Illinois 60115 \\ and Fermi National Accelerator Laboratory, P.O. Box 500, Batavia, Illinois 60510 \\ James D. Wells \\ CERN, Theory Division, CH-1211 Geneva 23, Switzerland
}

(Received 16 March 1999; published 7 July 1999)

\begin{abstract}
A Higgs boson with a mass below $150 \mathrm{GeV}$ has a total decay width of less than $20 \mathrm{MeV}$ into accessible standard model states. This narrow width means that the usual branching fractions for such a light Higgs boson are highly susceptible to any new particles to which it has unsuppressed couplings. In particular, there are many reasonable and interesting theoretical ideas that naturally imply an invisibly decaying Higgs boson. The motivations include models with light supersymmetric neutralinos, spontaneously broken lepton number, radiatively generated neutrino masses, additional singlet scalar(s), or right-handed neutrinos in the extra dimensions of TeV gravity. We discuss these approaches to model building and their implications for Higgs boson phenomenology in future Fermilab Tevatron runs. We find, for example, that the Tevatron with $30 \mathrm{fb}^{-1}$ integrated luminosity can make a $3 \sigma$ observation in the $l^{+} l^{-}+\mathbb{E}_{T}$ channel for a $125 \mathrm{GeV}$ Higgs boson that is produced with the same strength as the standard model Higgs boson but always decays invisibly. We also analyze the $b \bar{b}+\mathbb{E}_{T}$ final state signal and conclude that it is not as sensitive, but it may assist in excluding the possibility of an invisibly decaying Higgs boson or enable confirmation of an observed signal in the dilepton channel. We argue that a comprehensive Higgs boson search at the Tevatron should include the possibility that the Higgs boson decays invisibly. [S0556-2821(99)04815-8]
\end{abstract}

PACS number(s): 14.80.Cp, 12.60.Fr, 14.80.Bn

\section{INTRODUCTION}

The ultimate source of electroweak symmetry breaking (EWSB) is still mysterious. So far, progress in solving this mystery has been confined to ruling out ideas rather than confirming one. Nevertheless, an explanation exists and it is the primary purpose of the next generation colliders to find it.

We do know what the results of EWSB must be: the $W$ and $Z$ bosons must acquire mass, and the chiral fermions must acquire mass. The simplest explanation within the standard model (SM) is a scalar $S U(2)$ doublet which couples to the vector bosons via the covariant derivative and to the fermions via Yukawa couplings. After spontaneous symmetry breaking, one physical scalar degree of freedom remains the Higgs boson. Given all the other measurements that have already been made (gauge couplings and masses of the gauge bosons and fermions) the couplings of the Higgs boson to all SM particles are fixed, and the collider phenomenology is completely determined as a function of only one parameter, the Higgs boson mass.

The correct theory may be much different than our simplest notion. Low-energy supersymmetry, for example, is a rather mild deviation from the standard model EWSB idea. Nevertheless, supersymmetry requires at least two Higgs doublets that contribute to EWSB and complicate the phenomenology by having more parameters and more physical states in the spectrum. Furthermore, some theories, including supersymmetry, may allow other states with substantial couplings to exist which are light enough for a Higgs boson to decay into. EWSB burden sharing or Higgs boson interac- tions with other light states are in principle just as likely as the SM solution to EWSB.

In this paper we would like to add to the discussion of EWSB possibilities at a high luminosity Tevatron collider by considering a Higgs boson which decays invisibly. The SM Higgs boson case has been studied in great detail recently [1] for $\sqrt{s}=2 \mathrm{TeV}$ with high luminosity, and the prospects for discovering a light Higgs boson $\left(m_{h} \leqslant 130 \mathrm{GeV}\right)$ with $30 \mathrm{fb}^{-1}$ are promising [1]. Reaching beyond $130 \mathrm{GeV}$ will be more of a challenge, but studies in this direction appear tantalizing [2,3]. For an invisibly decaying Higgs boson, no studies have been performed to our knowledge. However, we believe it is interesting for many reasons.

The reason non-SM Higgs phenomena are especially relevant for the Tevatron is because at the Tevatron a Higgs boson is copiously produced only if its mass is less than 150 $\mathrm{GeV}$ or so. Such a light SM Higgs boson couples only very weakly to all on-shell decay-mode states, and has a narrow decay width in this range [4]. For example, $h \rightarrow f \bar{f}$ decays depend on the squared coupling $m_{f}^{2} / \mathrm{v}^{2}$, where $\mathrm{v}$ $=175 \mathrm{GeV}$. The largest mass fermion for a light Higgs boson to decay into is the $b$ quark with $m_{b} \simeq 4.5 \mathrm{GeV}$, leading to a squared coupling of order $m_{b}^{2} / \mathrm{v}^{2} \lesssim 10^{-3}$. As $m_{H}$ is increased above $135 \mathrm{GeV}$, the decays $H \rightarrow W W^{(*)}$ begin to become more important than $H \rightarrow b \bar{b}$. However, even for $m_{H}=(140,150) \mathrm{GeV}$, the total width of a SM Higgs boson is only about $(8,17) \mathrm{MeV}$. Therefore, if the light Higgs boson interacts with any new particle(s) in addition to the SM particles, the resulting impact on Higgs boson decay branching fractions could be dramatic. For example, an $\mathcal{O}(1)$ coupling of the Higgs boson to other light particles means that the 
Higgs boson will decay to these new states essentially $100 \%$ of the time. If the new states happen to not be detectable, none of the standard analyses for Higgs boson discovery would directly apply. ${ }^{1}$ Therefore, a comprehensive assessment of EWSB phenomenology at the Fermilab Tevatron must include considering the possibility of a Higgs boson decaying invisibly.

The current bounds on an invisibly decaying Higgs boson allow for a very interesting window to be explored at the Tevatron. At the CERN $e^{-} e^{+}$collider LEP II with $\sqrt{s}$ $=205 \mathrm{GeV}$, discovery should reach up to a mass of at least $95 \mathrm{GeV}$ [5]. At the CERN Large Hadron Collider (LHC), the discovery reach may be as high as $150 \mathrm{GeV}$ in the gauge process $p p \rightarrow Z \rightarrow Z H_{\text {inv }}[6,7]$ or $250 \mathrm{GeV}$ in the Yukawa process $p p \rightarrow t \bar{t} H_{\mathrm{inv}}[8]$. The current published limit is 80 $\mathrm{GeV}$ [9] from the $\sqrt{s}=184 \mathrm{GeV}$ run at LEP II. In the following sections we provide a motivation and analysis for the discovery of an invisibly decaying Higgs boson at the Tevatron run at high luminosity.

\section{MOTIVATION}

It follows from the above discussion that any theoretical idea which allows the light Higgs boson to interact with light invisible particles with $\mathcal{O}(1)$ couplings will result in $B(H$ $\rightarrow$ invisible $) \simeq 100 \%$. Many possibilities for this exist. In the following paragraphs we list a small subset of interesting theoretical ideas which could lead to a SM-like Higgs boson that decays invisibly.

\section{A. Higgs boson decays to neutralinos}

As a first example, the lightest supersymmetric partner (LSP), $\chi$, in supersymmetry may be a small mixture of Higgsino and $B$-ino (superpartners of the Higgs boson and hypercharge gauge boson), and so decays of the lightest Higgs boson into LSPs, $h \rightarrow \chi \chi$, may have a sizable probability. Or the LSP might be very nearly degenerate with other charged states which the Higgs boson decays into, and so decay products of the charged states are too soft to detect. If $R$ parity is conserved, the $\chi$ does not decay and escapes detection. Therefore, the Higgs boson is invisible. This possibility, however, is almost excluded for minimal supersymmetry based upon gauge coupling unification, gaugino mass unification, and scalar mass universality. In this case, the lightest neutralino is mostly a $B$-ino, and has mass approximately half that of the lightest chargino. The present bounds on the chargino are above about $90 \mathrm{GeV}$ [10], which in turn implies that the mass of the neutralino is at least $45 \mathrm{GeV}$ or so in minimal supersymmetry. Although it is possible to still

\footnotetext{
${ }^{1}$ In contrast, a heavy Higgs boson which is near or above the $W W$ threshold has a guaranteed decay mode with electroweak-strength coupling, and other unsuppressed decay modes enter at the $Z Z$ and $t \bar{t}$ thresholds. Therefore any new states which the Higgs boson may be allowed to decay into will likely not completely overwhelm the SM decay modes, so standard analyses will still be relevant for discovery at post-Tevatron colliders.
}

have $h \rightarrow \chi \chi$, the parameter space remaining for such decays has decreased and may continue to decrease if there is no discovery as the CERN LEP II $e^{+} e^{-}$collider runs proceed. Furthermore, in minimal supergravity parameter space the coupling of $\chi \chi h$ is often not significantly above that of $\bar{b} b h$ [11], and so $B(h \rightarrow \chi \chi) \gg B(h \rightarrow \bar{b} b)$ is not necessarily expected.

However, as we stray from the naive assumptions of gaugino mass unification and scalar mass universality, $h$ $\rightarrow \chi \chi$ is not as constrained by searches of chargino pair production at LEP II. Then the motivation is strengthened to consider the case where this branching ratio is high, leading to an invisibly decaying light Higgs boson. In supersymmetry with minimal particle content, the lightest Higgs boson is not expected to be above $125 \mathrm{GeV}$ [12]. We shall see later that the invisible Higgs boson can be probed with $3 \sigma$ significance up to about $125 \mathrm{GeV}$ at the Tevatron with $30 \mathrm{fb}^{-1}$.

\section{B. Higgs boson decays to neutrinos in extra dimensions}

Another interesting motivation is related to neutrino mass generation in theories with extra dimensions opening up at the $\mathrm{TeV}$ scale [13-15]. In this approach, which we will call "TeV gravity," no fundamental mass scale in field theory should exist above a few $\mathrm{TeV}$. Therefore, electroweak symmetry breaking, fermion masses, flavor dynamics, and neutrino masses all must occur near the TeV scale. The standard approach to neutrino mass generation is to introduce a righthanded neutrino and to apply a seesaw between a heavy Majorana mass of the neutrino $\left(m_{M}\right)$ and a rather light Dirac mass $\left(m_{D}\right)$. The lightest eigenvalue is then $m_{\nu}=m_{D}^{2} / m_{M}$. Typically, models prefer $m_{M} \gtrsim 10^{12} \mathrm{GeV}$ either because of naturalness or some considerations in $S O$ (10) model building, etc. In TeV gravity such high mass scales are not available.

It is mainly theoretical prejudice that has paradoxically made us consider extremely high mass scales to explain such low scales. One should not forget that there are many orders of magnitude between the neutrino mass and the weak scale in which nature could develop the right twist to explain itself. If $\mathrm{TeV}$ gravity is the correct approach to nature, then we must find the explanation and identify the phenomenology that can help us discern it. How this is related to the invisible Higgs boson will become apparent shortly.

If the right-handed neutrino is restricted to the SM 3-brane along with the other SM particles, neutrino masses would then need to be generated by dynamics near or below the $\mathrm{TeV}$ scale. There are viable alternatives for this, which may even lead to Higgs boson invisible decays [16]. However, one is enticed to postulate that the right-handed neutrino is free to propagate also in the extra dimensions where gravity propagates $[17,18]$. This is natural since $\bar{\nu}_{R}$ can be interpreted as a singlet that has no quantum numbers to restrict it to the SM brane. In this scenario, the $\bar{\nu}_{R}$ not only has its zero mode but Kaluza-Klein (KK) modes $\bar{\nu}_{R}^{(i)}$ separated in (mass) $)^{2}$ by $1 / R^{2}$ where $R$ is the linear dimension of the compact $\delta$ dimensions, determined from 


$$
M_{\mathrm{Pl}}^{2}=R^{\delta} M_{D}^{2+\delta}
$$

Here $\delta$ is the number of extra dimensions, $M_{\mathrm{Pl}}$ is the familiar Planck mass of the effective four-dimensional theory, and $M_{D} \sim 1 \mathrm{TeV}$ is the fundamental $D=4+\delta$ dimensional gravity scale. The absence of experimental deviations from Newtonian gravity at distances greater than $1 \mathrm{~mm}$ implies that $R \lesssim 10^{13} \mathrm{GeV}^{-1}$. For $\delta=1$, this implies $M_{D} \gtrsim 10^{9} \mathrm{GeV}$, but for $\delta \geqslant 2$ this does not impose any constraint stronger than $M_{D} \gtrsim 1 \mathrm{TeV}$.

Suppose that Dirac neutrino masses arise from Yukawa couplings $y_{\nu} H \bar{\nu}_{R} \nu_{L}$, so that $m_{\nu}=y_{\nu} \mathrm{v}$ where $\mathrm{v}=175 \mathrm{GeV}$ is the Higgs vacuum expectation value. Although the decay of $H$ to any given final state $\nu_{L} \bar{\nu}_{R}^{(i)}$ is proportional to $y_{\nu}^{2}$ and extremely small, the multiplicity of gauge-singlet righthanded neutrino KK states below $m_{H}$ can be very large. It is proportional to the volume $R^{\delta}$ of the $\delta$-dimensional space, with a momentum-space factor of order $m_{H}^{\delta}$ :

$$
\sum_{i} \rightarrow\left(m_{H} R\right)^{\delta}
$$

The total partial width of $H$ into $\mathrm{KK}$ excitations involving neutrinos is then of order

$$
\sum_{i} \Gamma\left(H \rightarrow \nu_{L} \bar{\nu}_{R}^{(i)}\right) \sim \frac{m_{H}}{16 \pi} y_{\nu}^{2}\left(m_{H} R\right)^{\delta} .
$$

Therefore the ratio of $\Sigma_{i} B\left(H \rightarrow \nu_{L} \bar{\nu}_{R}^{(i)}\right)$ to $B(H \rightarrow b \bar{b})$ can be estimated to be roughly

$$
x \equiv \frac{\sum_{i} B\left(H \rightarrow \nu_{L} \bar{\nu}_{R}^{(i)}\right)}{B(H \rightarrow \bar{b} b)} \simeq \frac{m_{\nu}^{2}}{3 m_{b}^{2}}\left(\frac{m_{H}}{M_{D}}\right)^{\delta}\left(\frac{M_{\mathrm{Pl}}}{M_{D}}\right)^{2}
$$

Now for $\delta=1$, the aforementioned constraint $M_{D} \gtrsim 10^{9} \mathrm{GeV}$ tells us that $x$ is negligibly small. For $\delta \geqslant 2$, there is no corresponding relevant constraint on $M_{D}$ and one can estimate

$$
x \simeq 10^{11-\delta}\left(\frac{m_{\nu}}{1 \mathrm{eV}}\right)^{2}\left(\frac{m_{H}}{100 \mathrm{GeV}}\right)^{\delta}\left(\frac{1 \mathrm{TeV}}{M_{D}}\right)^{2+\delta}
$$

The case $\delta=2$ may run into difficulties with nucleosynthesis, but for $\delta=3$, the decays into invisible states can dominate [17]. For example, with $m_{H} \gtrsim 100 \mathrm{GeV}$ one can have $x$ $\gtrsim 100$ even for $m_{\nu}^{2}=10^{-6} \mathrm{eV}^{2}$ and $M_{D}=1 \mathrm{TeV}$ or for $m_{\nu}^{2}$ $=10^{-1} \mathrm{eV}^{2}$ and $M_{D}=10 \mathrm{TeV}$. Larger values of $\delta$ can also give dominant invisible decays, although the estimate is increasingly sensitive to $M_{D}$. In any case, there is a strong possibility that the Higgs boson to KK neutrino partial width may greatly exceed the partial widths into SM states. Note also that there are no additional Higgs bosons necessary in this framework, allowing $\sigma(H Z)$ to occur at the same rate as $\sigma\left(H_{\mathrm{SM}} Z\right)$ in the SM.

\section{Higgs boson decays to Majorons}

Another approach is to assume that the traditional seesaw mechanism applies with a Majorana mass scale not much larger than $1 \mathrm{TeV}$. In this case, $m_{M}$ cannot be much bigger than about $1 \mathrm{TeV}$. If $m_{D} \sim 1 \mathrm{MeV}$ and $m_{M} \sim 1 \mathrm{TeV}$, then the neutrino mass is naturally $m_{\nu} \sim 1 \mathrm{eV}$. We leave it to model builders to decide why the Dirac mass of neutrinos may be near or below about $1 \mathrm{MeV}$. However, we remark that this is approximately the electron mass and so there is precedence in nature for a Dirac mass of a SM field to be near $1 \mathrm{MeV}$. That is, no extraordinary mass scales are required in the seesaw numerology of neutrino masses.

The question then centers on the origin of the Majorana mass. For us, the important consideration is whether the Majorana mass results from a spontaneously broken global symmetry. If $\eta$ is a singlet scalar field charged under a global lepton number and if $\eta$ couples to the neutrinos via the operator (in 2-component Weyl fermion notation) $\lambda \eta \bar{\nu}_{R} \bar{\nu}_{R}$, then a vacuum expectation value of $\eta$ will spontaneously break the global lepton number and generate a Majorana mass equal to $\lambda\langle\eta\rangle$. We can then identify $J=\operatorname{Im} \eta$ as the Nambu-Goldstone boson of the symmetry breaking $[19,20]$. It is easy to write down a potential between the SM Higgs doublet $\phi$ and the singlet scalar $\eta$, and to construct the interactions among mass eigenstates [21,22]. The two $C P$-even mass eigenstates are

$$
\begin{aligned}
& H=\cos \theta \operatorname{Re} \phi^{0}-\sin \theta \operatorname{Re} \eta \\
& S=\sin \theta \operatorname{Re} \phi^{0}+\cos \theta \operatorname{Re} \eta .
\end{aligned}
$$

The partial widths of $H \rightarrow J J$ and $H \rightarrow b \bar{b}$ can be calculated in an arbitrary potential $V=V\left(\phi^{\dagger} \phi, \eta^{\dagger} \eta\right)$ consistent with gauge invariance and global lepton number invariance. The ratio of these partial widths [21] (branching fractions) can then be expressed as

$$
x \equiv \frac{B(H \rightarrow J J)}{B(H \rightarrow \bar{b} b)} \simeq \frac{\tan ^{2} \theta}{12}\left(\frac{m_{H}}{m_{b}}\right)^{2} \frac{\langle\phi\rangle^{2}}{\langle\eta\rangle^{2}} .
$$

There are several consequences to notice from Eq. (8). First, if $\langle\eta\rangle \gg\langle\phi\rangle$ or, equivalently, if $m_{M} \gg m_{Z}$, the Higgs boson decays into $J J$ would not happen very often. In the usual discussion of the Majoron model approach to neutrino masses the prospect of $m_{M} \sim m_{Z}$ is just one possibility over a very wide range of choices for $m_{M}$. However, in TeV gravity, for example, it is required that $m_{M}$ cannot be higher than the weak scale, leading to a potentially large branching fraction of $H \rightarrow J J$. The second point to notice in Eq. (8) is implicit. If $\tan \theta \gg 1$, then $B(H \rightarrow J J) \rightarrow 100 \%$. However, in this case $\sigma(H Z)$ is proportional to $\cos ^{2} \theta \rightarrow 0$, because the $H Z Z$ coupling scales with $\cos \theta$. In reality the invisible Higgs rate in this model for $m_{Z} \lesssim m_{H} \lesssim 150 \mathrm{GeV}$ is

$$
\frac{\sigma(Z H \rightarrow Z+J J)}{\sigma\left(Z H_{\mathrm{SM}}\right)}=\xi(x, \cos \theta) \frac{x}{1+x} \cos ^{2} \theta
$$


where $\xi \simeq 1$ represents a small correction from $H$ $\rightarrow W W^{*}, \tau \tau$ decays for $m_{H} \lesssim 130 \mathrm{GeV}$ and a more sizable $\xi \lesssim 1$ correction for larger Higgs boson mass values.

Therefore, it is impossible in this approach to have $\sigma(Z$ $+J J)>\sigma\left(Z H_{\mathrm{SM}}\right)$. Nevertheless, it is quite possible and natural for $\sigma(Z+H \rightarrow Z+J J)$ to be the dominant production and decay mode of $H$, and to have a production cross section close to the value of a SM Higgs boson of the same mass.

\section{Standard model with an extra singlet}

There are many variations on the above themes which will have impact on the production rate of the relevant Higgs boson and its decays into invisible particles. Rather than trying to parametrize all the possibilities with complicated formulas, we instead choose to study an equally motivated but simpler model such that one can scale the results to any other particular idea. In this model there exists one gauge-singlet scalar boson and one doublet Higgs boson whose vacuum expectation value constitutes all of the EWSB and which therefore couples to the $W$ and $Z$ bosons with the same strength as the SM Higgs boson. This minimal extension, as we will see, has a strong impact on the invisible width of the Higgs boson [23,24].

When one adds a SM singlet to the spectrum, the full Lagrangian becomes

$$
\mathcal{L}=\mathcal{L}_{\mathrm{SM}}-m_{S}^{2}|S|^{2}-\lambda^{\prime}|S|^{2}|H|^{2}-\lambda^{\prime \prime}|S|^{4},
$$

where $H$ is the SM doublet Higgs boson and $S=S^{0}+i A_{S}^{0}$ is the complex singlet Higgs boson. In writing Eq. (10), we have assumed only that $S$ is charged under a $U(1)_{S}$ global symmetry and that the Lagrangian respects this symmetry. Without this symmetry one could write down more terms, such as $\left(S^{* 2}+S^{2}\right)|H|^{2}$, but these do not qualitatively change the discussion below. Now if $\langle S\rangle \neq 0$, the model is the same as the Majoron model discussed earlier, with $U(1)_{S}$ playing the role of lepton number.

If $\langle S\rangle=0$, there is no mixing between the $S$ and the $H$, and if $m_{S}<m_{H} / 2$, then $H \rightarrow S^{0} S^{0}, A_{S}^{0} A_{S}^{0}$ are allowed to proceed with coupling $\mu_{S}=2 \lambda^{\prime}\langle H\rangle$. If $\mu_{S} \gg m_{b}$, these decays will be near $100 \%$ for a light Higgs boson mass below about $150 \mathrm{GeV}$. Since the $S^{0}$ does not mix with the $H$, there will be no suppression of $\mathrm{ZH}$ production. Also, since $S$ has no couplings to SM gauge bosons or fermions, it will be stable and non-interacting (invisible) in the detectors. For the remainder of this paper we assume this model where $\sigma(Z H)$ is unsuppressed compared to the SM and $H \rightarrow$ invisible with $100 \%$ branching fraction. One can then scale the results to other, more complicated models which may have suppressions in the total production cross section or in the invisible decay width. One should keep in mind that the optimal experimental analysis will be to combine search results over all channels, including invisible decay products, $b \bar{b}, \tau^{+} \tau^{-}$, etc., to search for evidence in the data of a scalar Higgs boson that may decay to several final states with similar probabilities.

\section{DETECTING AN INVISIBLY DECAYING HIGGS BOSON WITH LEPTONS}

The process we have found most significant in the search for an invisibly decaying Higgs boson in $p \bar{p}$ collisions at the Tevatron with $\sqrt{s}=2 \mathrm{TeV}$ is

$$
p \bar{p} \rightarrow Z^{*} \rightarrow\left(Z \rightarrow l^{+} l^{-}\right)\left(H_{\mathrm{inv}} \rightarrow \text { invisible }\right) .
$$

The signal is therefore two oppositely charged same-flavor leptons with invariant mass $m_{Z}$, accompanied by missing transverse energy from the invisibly decaying Higgs boson. By $l^{+} l^{-}$we mean $e^{+} e^{-}$or $\mu^{+} \mu^{-}$and not $\tau^{+} \tau^{-}$. The $\tau^{+} \tau^{-}$ final states may be used to gain in significance slightly, but the uncertainties in $\tau$ identification and invariant mass resolution lead us to ignore this final state in the present analysis. Again, we are assuming a theory which is identical to the SM except that a light singlet scalar model exists that the Higgs boson can decay into. As discussed in the previous section, this model then implies that $\sigma\left(H_{\text {inv }} Z\right)=\sigma\left(H_{\mathrm{SM}} Z\right)$ and $B\left(H_{\text {inv }} \rightarrow\right.$ invisible $) \simeq 100 \%$.

The most important background is $Z Z$ production where one $Z$ boson decays leptonically and the other $Z$ boson decays into neutrinos. Since $Z Z$ is produced by $t$-channel processes, it is expected that the $E_{T}$ distribution of the $Z$ bosons will be softer (lower energy) than the $E_{T}$ distribution of the $Z$ boson accompanying $Z H_{\text {inv }}, s$-channel production. An equivalent statement at leading order (also NLO with a jet veto) is that the missing transverse energy in the $Z Z$ background will typically be smaller than the missing energy distribution in $Z_{\text {inv }}$ events for Higgs bosons with mass near $m_{Z}$.

The next most significant background is from $W^{+} W^{-}$ production with each $W$ decaying leptonically. (We have included here contributions from $W \rightarrow \tau \nu$ followed by a leptonic $\tau$ decay.) This background has a considerably softer transverse energy distribution. As we will see in the plots and discussion below, the fact that both of the leading backgrounds have softer transverse energy profiles than the signal allows the possibility to gain significance by choosing a high cut on $\boldsymbol{E}_{T}$. Finite detector resolution and smearing effects may also favor choosing a higher $\boldsymbol{E}_{T}$ cut. However, if the lower bound on $\mathbb{E}_{T}$ is chosen to be too high, then one will simply run out of signal. Therefore some intermediate choice of cut for $\mathbb{E}_{T}$ is required.

Other important backgrounds to consider arise from $W Z$, $W j$, and $Z^{(*)} \rightarrow \tau^{+} \tau^{-} \rightarrow l^{+} l^{-}+\mathbb{E}_{T}$. The $Z^{(*)} \rightarrow \tau^{+} \tau^{-}$background is made completely negligible by requiring that $m_{l^{+} l^{-}} \simeq m_{Z}, \boldsymbol{E}_{T}>50 \mathrm{GeV}$, and $\cos \left(\phi_{l^{+} l^{-}}\right)>-0.9$. The angle $\phi_{l^{+} l^{-}}$is the angle between the two leptons in the transverse plane. The $W Z$ background requires that a lepton from $W$ $\rightarrow l \nu$ not be detected. This has a rather low probability, and our analysis requires that the pseudo-rapidity of the missed lepton be above $|\eta|>2$. The $W j$ background can mimic the signal final state if the jet registers in the detector as a lepton of the right flavor and charge to partner with the lepton from $W \rightarrow l \nu$. We liberally put this fake rate of $j \rightarrow l$ at $10^{-4}$. Other backgrounds from grossly mismeasured jet energies, $W Z$ production with $W \rightarrow \tau \nu$, and $t \bar{t}$ production can be elimi- 
nated by vetoing events with a jet with transverse energy greater than $10 \mathrm{GeV}$ and $|\eta|<2.5$.

We now summarize all the kinematic cuts applied in this analysis:

$$
\begin{gathered}
p_{T}\left(l^{+}\right), \quad p_{T}\left(l^{-}\right)>12 \mathrm{GeV} \\
\left|\eta\left(l^{+}\right)\right|<2, \quad\left|\eta\left(l^{-}\right)\right|<2 \\
\left|m_{l^{+} l^{-}}-m_{Z}\right|<7 \mathrm{GeV} \\
\cos \left(\phi_{l^{+} l^{-}}\right)>-0.9 \\
\boldsymbol{E}_{T}>50 \mathrm{GeV} .
\end{gathered}
$$

The actual analysis of signals and backgrounds was carried out at the parton level using the COMPHEP program [25], except for the $W W \rightarrow \tau l \nu \bar{\nu} \rightarrow l l+\boldsymbol{E}_{T}$, which was included using the ISAJET [26] Monte Carlo program. We also summarize some relevant detector parameters that we assume:

$$
\begin{gathered}
\operatorname{probability}(j \rightarrow l)=10^{-4} \\
\text { lost lepton has }|\eta(l)|>2 \\
\text { dilepton id efficiency in } Z \rightarrow l^{+} l^{-}=0.7 \\
\text { NLO } K \text { factor } \times \text { jet veto }=\mathrm{LO} .
\end{gathered}
$$

The dilepton identification rate is taken from [27]. The last line refers to the fact that NLO calculations of EW gauge boson production $V V^{\prime}$ and gauge boson with Higgs boson production $V H$ has a $K$ factor of slightly less than $1.4[28,29]$ at the Tevatron. The jet veto efficiency assuming that jets must have $p_{T}>10 \mathrm{GeV}$ and $\left|\eta_{j}\right|<2.5$ is approximately $70 \%$ [27]. Multiplying these two numbers together gives 1.4 $\times 0.7 \simeq 1$, which is what we assume for the analysis. This is equivalent to simulating background and signal at leading order (LO). Loosening the jet veto requirement somewhat might lead to a slightly larger significance.

In Figs. 1 and 2 we plot the dilepton $E_{T}$ (equivalent to $\boldsymbol{E}_{T}$ ) spectrum for the background and signal for various Higgs boson masses. As expected, the $Z Z$ and $W W$ backgrounds are the most significant, and the other backgrounds are down significantly from them. Moreover, the $W W$ background is reduced quite significantly by choosing a higher $E_{T}$ cut. Results for the cross sections after cuts and efficiencies are given, for the $m_{H}=100$ and 130 signals and the total background for different choices of the $E_{T}$ cut, in Table I.

Using the definition

$$
\text { significance }=S / \sqrt{B}
$$

where $S$ and $B$ are the signal and background in $\mathrm{fb}$, we plot the significance of the signal compared to background in Fig. 3 as a function of $E_{T}$. The peak of the significance curve occurs at different $E_{T}$ depending on the mass of the Higgs boson. For larger masses the significance peak is at larger $E_{T}$. This is expected since heavier Higgs bosons will tend to carry away more missing energy and be accompanied by

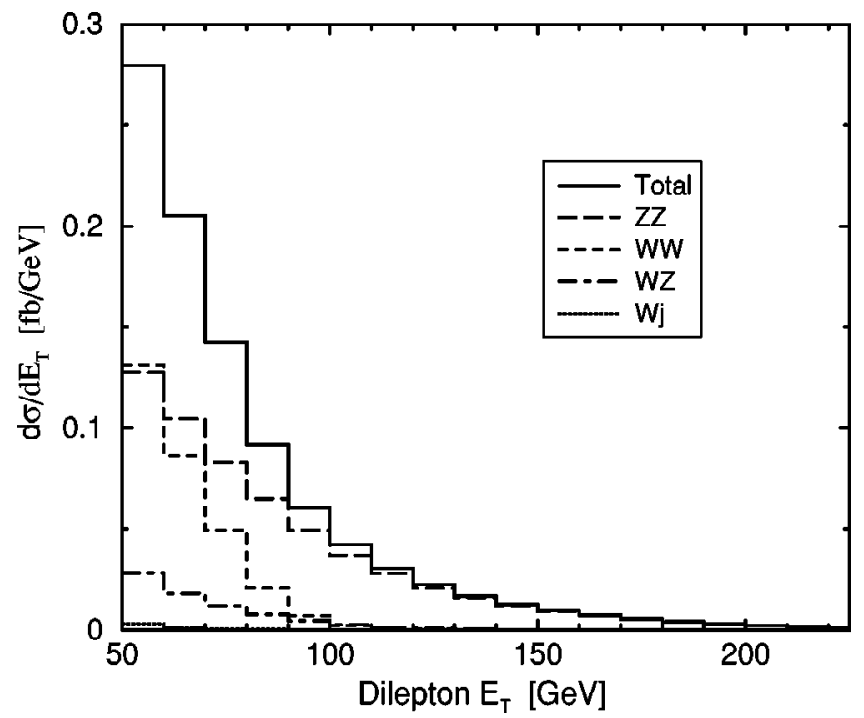

FIG. 1. The distribution of the backgrounds for $l^{+} l^{-}+\mathbb{E}_{T}$ as a function of the dilepton $E_{T}$ or, equivalently, $\mathbb{E}_{T}$. This distribution is plotted after all cuts and efficiencies have been applied except the cut on $E_{T}$.

more boosted $Z$ bosons, and because the $W W$ component of the background has a much softer $E_{T}$ distribution. In our analysis we choose the $E_{T}$ cut for each Higgs mass in order to maximize the significance, although the significance is a rather flat function of this cut.

We are now in position to predict how much luminosity is required at the Tevatron to produce a 95\% $(1.96 \sigma)$ exclusion limit, a $3 \sigma$ observation, and a $5 \sigma$ discovery [30]. The results are shown in Fig. 4 and Table II. If we assume that the Tevatron will accumulate a total of $30 \mathrm{fb}^{-1}$ of integrated luminosity, the invisible Higgs bosons could be excluded at the $95 \%$ confidence level up for $m_{H}$ up to nearly $150 \mathrm{GeV}$.

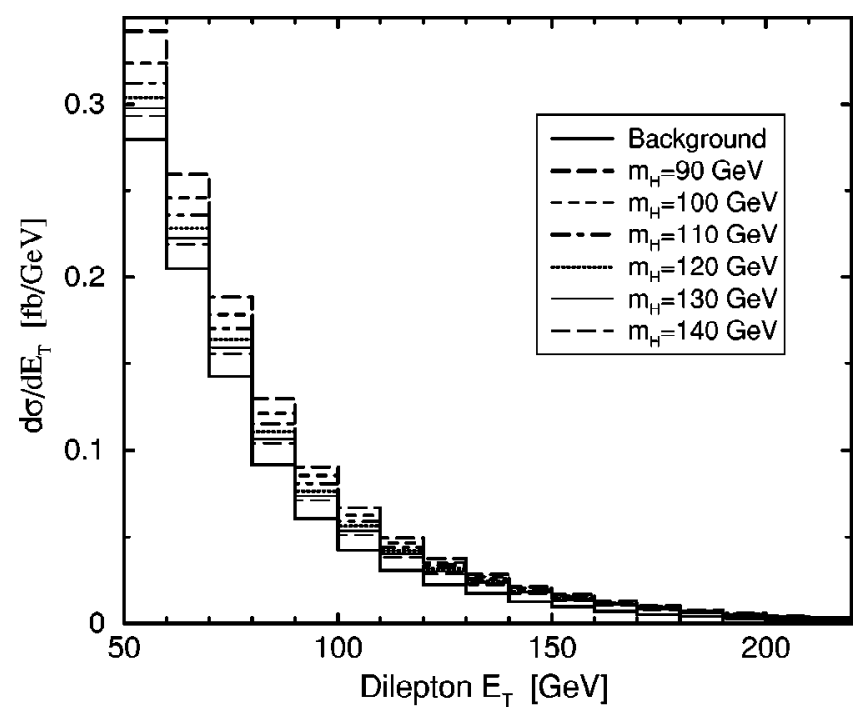

FIG. 2. The distribution of the background and signal + background for $\mathrm{ZH}_{\mathrm{inv}} \rightarrow \mathrm{l}^{+} l^{-}+\boldsymbol{E}_{T}$ as a function of the dilepton $E_{T}$ or, equivalently, $\boldsymbol{E}_{T}$. This distribution is plotted after all cuts and efficiencies have been applied except the cut on $E_{T}$. 
TABLE I. Cross sections after cuts and efficiencies for $m_{H}$ $=100$ and $130 \mathrm{GeV}$ and the total background for various choices of the $E_{T}$ cut. All cuts and efficiencies except for the $E_{T}$ cut have been applied.

\begin{tabular}{cccc}
\hline \hline $\begin{array}{r}E_{T} \text { cut } \\
{[\mathrm{GeV}]}\end{array}$ & $\begin{array}{c}m_{H}=100 \mathrm{GeV} \\
\text { signal }[\mathrm{fb}]\end{array}$ & $\begin{array}{c}m_{H}=130 \mathrm{GeV} \\
\text { signal }[\mathrm{fb}]\end{array}$ & Background \\
\hline 50 & 2.71 & 1.40 & 9.44 \\
60 & 2.26 & 1.22 & 6.65 \\
70 & 1.86 & 1.05 & 4.60 \\
80 & 1.50 & 0.88 & 3.17 \\
90 & 1.20 & 0.73 & 2.25 \\
100 & 0.96 & 0.60 & 1.64 \\
110 & 0.76 & 0.49 & 1.22 \\
120 & 0.60 & 0.40 & 0.91 \\
\hline \hline
\end{tabular}

(Note, however, that the theoretical motivation for an invisibly decaying Higgs boson is reduced anyway as $m_{H}$ increases above $150 \mathrm{GeV}$ and the $H \rightarrow W W^{(*)}$ mode opens up.) A $3 \sigma$ observation is possible for masses up to approximately $125 \mathrm{GeV}$, and a $5 \sigma$ discovery is not possible for $m_{H_{\text {inv }}}$ $>100 \mathrm{GeV}$. This should be compared with LEP II with $\sqrt{s}$ $=205 \mathrm{GeV}$ which should be able to discover $H_{\text {inv }}$ if its mass is below $95 \mathrm{GeV}$ [5]. The current limit on $m_{H_{\text {inv }}}$ from $\sqrt{s}$ $=184 \mathrm{GeV}$ data at LEP II is $80 \mathrm{GeV}$ [9].

Our results have been based only on counting events with $E_{T}$ larger than some cut. After detector responses have been more firmly established, it may also be worth investigating whether the shape of the $E_{T}$ distribution, compared to the expected background profile, can be employed to exclude or substantiate a signal. In effect, the plentiful $l^{+} l^{-}+\mathbb{E}_{T}$ events with smaller $\boldsymbol{E}_{T}$ (even less than $50 \mathrm{GeV}$ ) could be used to get a handle on background levels which can then be tested with the higher $\boldsymbol{E}_{T}$ events where the signal has its main support.

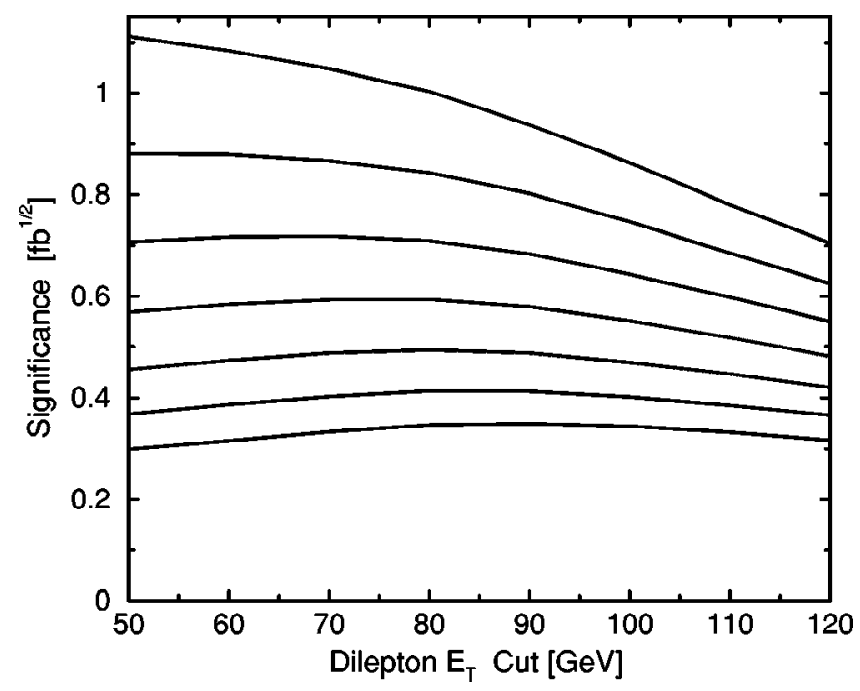

FIG. 3. Calculation of the significance in $\mathrm{fb}^{1 / 2}$, defined as $S / \sqrt{B}$, as a function of the dilepton $E_{T}$ or, equivalently, $\boldsymbol{E}_{T}$. The lines from top to bottom refer to $m_{H_{\text {inv }}}=90,100,110,120,130,140,150$ $\mathrm{GeV}$.

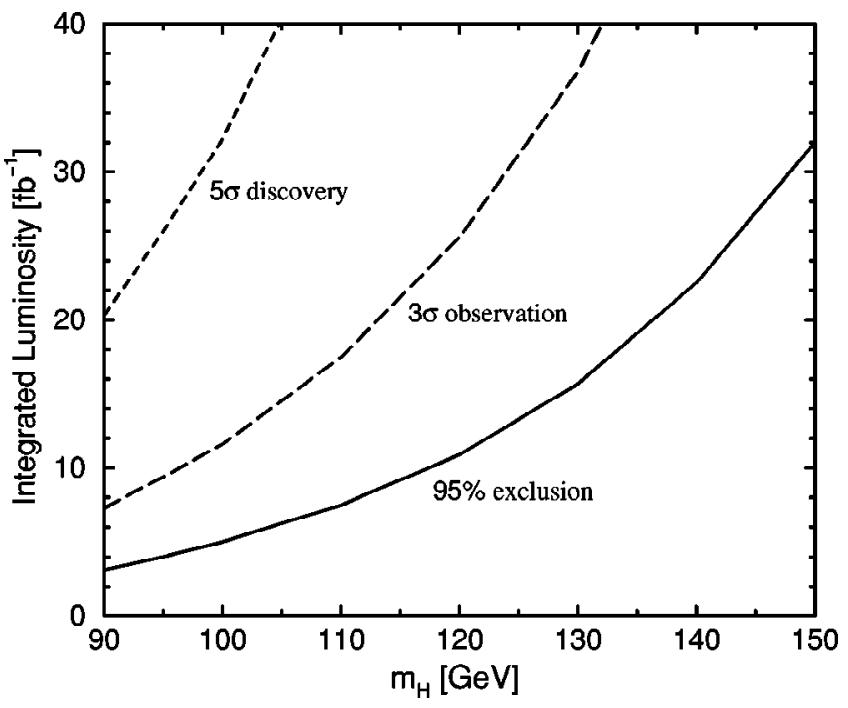

FIG. 4. Contours of $95 \%$ exclusion, $3 \sigma$ observation, and $5 \sigma$ discovery in the $m_{H_{\text {inv }}}$ vs luminosity plane. From this plot one can learn, for example, that with $30 \mathrm{fb}^{-1}$ a $3 \sigma$ observation is possible for $m_{H_{\text {inv }}} \lesssim 125 \mathrm{GeV}$.

This could be done, for example, using an optimized neural net procedure.

\section{DETECTING THE INVISIBLY DECAYING HIGGS BOSON WITH $b$ QUARKS}

Another signal that is potentially useful for discovering an invisibly decaying Higgs boson is ${ }^{2}$

$$
p \bar{p} \rightarrow(Z \rightarrow b \bar{b})\left(H_{\mathrm{inv}} \rightarrow \text { invisible }\right)=b \bar{b}+\boldsymbol{E}_{T} .
$$

The advantage of this signal is the increased branching fraction of $Z \rightarrow b \bar{b}$ compared to $Z \rightarrow l^{+} l^{-}$. The disadvantages are the lower efficiency for identifying $b \bar{b}$ final states compared to leptonic final states, the reduced invariant mass resolution of $Z \rightarrow b \bar{b}$, and more difficult background sources.

The signal of Eq. (22) is very similar to a $b \bar{b}+\mathbb{E}_{T}$ signal accessible in the SM [31-33]:

$$
p \bar{p} \rightarrow(Z \rightarrow \nu \bar{\nu})\left(H_{\mathrm{SM}} \rightarrow b \bar{b}\right)=b \bar{b}+\mathbb{E}_{T}
$$

Therefore, we can directly apply the background studies of this complementary signal to the invisibly decaying Higgs boson signal. In Ref. [32] the signal and backgrounds for $b \bar{b}+\boldsymbol{E}_{T}$ were studied using the following cuts and efficiency parameters:

$$
\begin{gathered}
p_{T}\left(b_{1}\right), p_{T}\left(b_{2}\right)>20 \mathrm{GeV}, 15 \mathrm{GeV} \\
\left|\eta\left(b_{1,2}\right)\right|<2
\end{gathered}
$$

\footnotetext{
${ }^{2}$ Other possible signals involving $p \bar{p} \rightarrow Z H$ followed by $Z \rightarrow j j$ without tagged $b$ jets will suffer from large backgrounds due to multiple partonic contributions to $p \bar{p} \rightarrow j j Z \rightarrow j j \nu \bar{\nu}$.
} 
TABLE II. Luminosity required to make a $95 \%$ confidence level exclusion, $3 \sigma$ observation, and $5 \sigma$ discovery of an invisibly decaying Higgs boson in the $\mathrm{ZH}_{\mathrm{inv}} \rightarrow l^{+} l^{-}+\boldsymbol{E}_{T}$ channel.

\begin{tabular}{cccc}
\hline \hline$m_{H_{\text {inv }}}[\mathrm{GeV}]$ & $\begin{array}{c}95 \% \text { exclusion } \\
\text { luminosity }\left[\mathrm{fb}^{-1}\right]\end{array}$ & $\begin{array}{c}3 \sigma \text { observation } \\
\text { luminosity }\left[\mathrm{fb}^{-1}\right]\end{array}$ & $\begin{array}{c}5 \sigma \text { discovery } \\
\text { luminosity }\left[\mathrm{fb}^{-1}\right]\end{array}$ \\
\hline 90 & 3.1 & 7.3 & 20 \\
100 & 5.0 & 11.6 & 32 \\
110 & 7.5 & 17.5 & 49 \\
120 & 10.9 & 26 & 71 \\
130 & 15.7 & 37 & 103 \\
140 & 23 & 53 & 206 \\
150 & 32 & 74 & \\
\hline \hline
\end{tabular}

$\phi\left(b_{1}, \boldsymbol{E}_{T}\right), \phi\left(b_{2}, \boldsymbol{E}_{T}\right)>0.5 \mathrm{rad}$

$$
H_{T} \equiv \sum E_{T}(j)<175 \mathrm{GeV}
$$

$$
\boldsymbol{E}_{T}>35 \mathrm{GeV}
$$

$70 \mathrm{GeV}<m_{b b}<110 \mathrm{GeV} \quad$ (loose cut)

$$
80 \mathrm{GeV}<m_{b b}<100 \mathrm{GeV} \quad \text { (tight cut) }
$$

$$
Z \rightarrow b \bar{b} \text { efficiency }=0.49(70 \% \text { for each } b)
$$

The cut on $\phi\left(b, \mathbb{E}_{T}\right)$ is to ensure that the missing energy does not originate from a grossly mismeasured $b$ jet, which may, for example, have neutrino(s) carrying away much of its energy. We will also present results based on the assumption of "loose" $m_{b b}$ invariant mass resolution and on the "tight" $m_{b b}$ invariant mass resolution as indicated in the above. The $b \bar{b}+\mathbb{E}_{T}$ total background after all cuts are applied is $51.1 \mathrm{fb}$ for the "loose" $m_{b b}$ resolution and $32.3 \mathrm{fb}$ for the "tight" $m_{b b}$ resolution [32]. These background totals include contributions from $Z Z, W Z, Z b \bar{b}, W b \bar{b}$, single top quark, and $t \bar{t}$ production.
To apply these background studies to the present invisibly decaying Higgs boson situation, we simulate the signal given the same kinematic cuts and efficiency parameters. Our simulation is at the parton level, and so we must further take into account realistic $b$-jet energy corrections and jet reconstruction. Also, $H_{T}$ is simply the sum of the two $b$-jet energies in our parton-level computations, but in the analysis of Refs. [31,32] it includes a sum over other jets as well. To take these factors into account, we can take advantage of the fact that, for $m_{H}=m_{Z}$, the two signals are exactly the same except for the known effects of branching fractions $H, Z$ $\rightarrow b \bar{b}$ and $Z \rightarrow \nu \bar{\nu}$. Therefore we normalize our total efficiency for the $m_{H_{\text {inv }}}=m_{Z}$ case to be equal to the efficiency found in [32] for the $m_{H_{S M}}=m_{Z}$ case. Since our signal always has $Z \rightarrow b \bar{b}$, we can apply this overall normalization efficiency factor for all values of $m_{H_{\text {inv }}}$ with little error. A dedicated analysis of $b \bar{b}$ efficiencies as a function of $H_{\text {inv }}$ would likely indicate a slight increase in efficiency since the $Z$ boson $p_{T}$ and, therefore, the average $b$-jet $p_{T}$ values increase as $m_{H_{\text {inv }}}$ increases. Furthermore, the missing transverse energy will systematically increase with $m_{H_{\text {inv }}}$, allowing for events to pass the missing energy cut with less sensitivity to $b$-jet energy fluctuations around their intrinsic parton values. It is quite possible that the significance can be

TABLE III. Signal and significance results for the $Z H_{\text {inv }} \rightarrow b \bar{b}+\boldsymbol{E}_{T}$ process at $\sqrt{s}=2 \mathrm{TeV}$ Tevatron, estimated after cuts and efficiencies as described in the text. The "loose" column refers to assuming $70 \mathrm{GeV}<m_{b b}<110 \mathrm{GeV}$ invariant mass resolution, and the "tight" column refers to assuming $80 \mathrm{GeV}$ $<m_{b b}<100 \mathrm{GeV}$ invariant mass resolution. The final column is the required luminosity to reach a $95 \%$ exclusion with the assumption of "tight" $b b$ invariant mass resolution. With $30 \mathrm{fb}^{-1}$, therefore, a $95 \%$ exclusion could be obtained for $m_{H_{\text {inv }}} \lesssim 115 \mathrm{GeV}$.

\begin{tabular}{ccccc}
\hline \hline$m_{H_{\text {inv }}}[\mathrm{GeV}]$ & $\sigma\left(b \bar{b}+\boldsymbol{E}_{T}\right)[\mathrm{fb}]$ & $\begin{array}{c}S / \sqrt{B}[\sqrt{\mathrm{fb}}] \\
\text { "loose" }\end{array}$ & $\begin{array}{c}S / \sqrt{B}[\sqrt{\mathrm{fb}}] \\
\text { "tight” }\end{array}$ & $\begin{array}{c}95 \% \text { exclusion } \\
\text { luminosity }\left[\mathrm{fb}^{-1}\right]\end{array}$ \\
\hline 90 & 4.10 & 0.57 & 0.72 & 7.4 \\
100 & 3.13 & 0.44 & 0.55 & 12.7 \\
110 & 2.41 & 0.34 & 0.42 & 22 \\
120 & 1.87 & 0.26 & 0.33 & 35 \\
130 & 1.46 & 0.20 & 0.26 & 57 \\
140 & 1.15 & 0.16 & 0.20 & 150 \\
150 & 0.91 & 0.13 & 0.16 & \\
\hline \hline
\end{tabular}


increased somewhat by raising the $\mathbb{E}_{T}$ cut to take advantage of this. We therefore conclude that our approach is justified, and perhaps yields slightly too pessimistic results.

In Table III we list the signal cross section after cuts and efficiencies and the significance for the $b \bar{b}+\boldsymbol{E}_{T}$ signal. The last column is the required luminosity to make a $95 \%$ exclusion of the invisibly decaying Higgs boson based upon the $b \bar{b}+\mathbb{E}_{T}$ final state and the "tight" $m_{b b}$ invariant mass resolution. With $30 \mathrm{fb}^{-1}, m_{H_{\text {inv }}} \leq 115 \mathrm{GeV}$ could be excluded. With the same luminosity, a $3 \sigma$ observation could be made for $m_{H_{\text {inv }}} \lesssim 100 \mathrm{GeV}$; however, most or all of this region will likely be probed earlier by the CERN LEP II $e^{+} e^{-}$collider. We can clearly see that at the present time the significance of this channel in discovering the light invisible Higgs boson is not as high as in the $l^{+} l^{-}+\boldsymbol{E}_{T}$ channel. Nevertheless, $b \bar{b}$ $+\mathbb{E}_{T}$ could be a useful channel to combine with $l^{+} l^{-}+\boldsymbol{E}_{T}$ to investigate exclusion ranges and also to obtain confirmation of an observed signal if an excess were to develop.

\section{CONCLUSION}

In summary, there are many reasonable theoretical ideas which lead to a light Higgs boson that most often decays invisibly. Several of these ideas, including Higgs decays to Majorons or right-handed neutrinos, are made possible by mechanisms which generate neutrino masses. Thus, our ignorance of neutrino mass generation is correlated with our ignorance of how likely Higgs bosons will decay invisibly. Experimentally, no theoretical prejudices should prevent the search for this possibility. This is especially important at the Tevatron, since low mass Higgs bosons have very weak SM couplings, and so any non-standard coupling of the Higgs boson to other particles will likely garner a significant branching fraction, perhaps even near 100\%. Higgs bosons with mass much above about $150 \mathrm{GeV}$ are not likely to be completely invisible since SM couplings to the EWSB Higgs boson exist which are $\mathcal{O}(1)$ in strength, and thus lead to visible decay modes.

The experimental search capability of an invisible Higgs bosons at the Tevatron requires non-SM search strategies outlined in the previous sections. With $30 \mathrm{fb}^{-1}$ one could observe (at $3 \sigma$ ) an invisible Higgs boson with mass up to approximately $125 \mathrm{GeV}$ in the $l^{+} l^{-}+\boldsymbol{E}_{T}$ channel and up to $100 \mathrm{GeV}$ in the $b \bar{b}+\mathbb{E}_{T}$ channel. It should be noted that the presence or absence of an excess in these channels will require knowledge of backgrounds which come primarily from $Z Z$ and $W W$. The total rates for these processes will be difficult to model with great accuracy. However, they can be measured directly by observation of other final states, e.g. $p \bar{p} \rightarrow Z Z \rightarrow l^{+} l^{-} b \bar{b}$ and the rarer but clean $p \bar{p} \rightarrow Z Z$ $\rightarrow l^{+} l^{-} l^{\prime} l^{\prime}$, as well as $l^{+} l^{-}+\boldsymbol{E}_{T}$ events with lower $\boldsymbol{E}_{T}$. The fact that these backgrounds will need to be well understood is a general feature of Higgs boson searches, and is not strictly limited to the invisibly decaying Higgs boson search. Nevertheless, an opportunity exists for a high-luminosity Tevatron to discover or exclude the invisibly decaying Higgs boson in the low mass region, which is the most likely place where an invisible Higgs boson would reside.

\section{ACKNOWLEDGMENTS} sions.

We thank D. Hedin and A. Pilaftsis for helpful discus-
[1] Proceedings of the Run II Workshop on Higgs/ Supersymmetry, Fermilab publication to appear. Preliminary results available at http://fnth37.fnal.gov/higgs.html

[2] H. Baer and J.D. Wells, Phys. Rev. D 57, 4446 (1998).

[3] T. Han, A. Turcot, and R.-J. Zhang, Phys. Rev. D 59, 093001 (1999).

[4] See, for example, M. Spira, A. Djouadi, D. Graudenz, and P.M. Zerwas, Nucl. Phys. B453, 17 (1995).

[5] B. Brahmachari et al., Phys. Rev. D 48, 4224 (1993); F. de Campos, O.J.P. Eboli, J. Rosiek, and J.W.F. Valle, ibid. 55, 1316 (1997).

[6] D. Choudhury and D.P. Roy, Phys. Lett. B 322, 368 (1994).

[7] S. Frederiksen, N. Johnson, G. Kane, and J. Reid, Phys. Rev. D 50, R4244 (1994).

[8] J. Gunion, Phys. Rev. Lett. 72, 199 (1994).

[9] ALEPH Collaboration, R. Barate et al., Phys. Lett. B 450, 301 (1999); L3 Collaboration, M. Acciarri et al., ibid. 418, 389 (1998); OPAL Collaboration, K. Ackerstaff et al., ibid. 393, 231 (1997).

[10] DELPHI Collaboration, P. Abreu et al., Phys. Lett. B 446, 75 (1999); L3 Collaboration, M. Acciarri et al., Eur. Phys. J. C 4, 207 (1998); ALEPH Collaboration, R. Barate et al., Report
No. CERN-EP/99-014; OPAL Collaboration, G. Abbiendi et al., Eur. Phys. J. C 8, 255 (1999).

[11] G.L. Kane, G.D Kribs, S.P. Martin, and J.D. Wells, Phys. Rev. D 53, 213 (1996).

[12] K. Matchev and D. Pierce, Phys. Lett. B 445, 331 (1999).

[13] N. Arkani-Hamed, S. Dimopoulos, and G. Dvali, Phys. Lett. B 429, 263 (1998); I. Antoniadis, N. Arkani-Hamed, S. Dimopoulos, and G. Dvali, ibid. 436, 257 (1998).

[14] I. Antoniadis, Phys. Lett. B 246, 377 (1990); I. Antoniadis, C. Munoz, and M. Quiros, Nucl. Phys. B397, 515 (1993). J.D. Lykken, Phys. Rev. D 54, 3693 (1996).

[15] K. Dienes, E. Dudas, and T. Gherghetta, Phys. Lett. B 436, 55 (1998); Nucl. Phys. B537, 47 (1999).

[16] For example, see A. Pilaftsis, Z. Phys. C 55, 275 (1992). Here, neutrino masses generated radiatively below the $\mathrm{TeV}$ scale may lead to $H \rightarrow \nu_{\text {light }} \bar{\nu}_{\text {heavy }}$ invisible decays.

[17] N. Arkani-Hamed, S. Dimopoulos, G. Dvali, and J. MarchRussell, hep-ph/9811448.

[18] K. Dienes, E. Dudas, and T. Gherghetta, hep-ph/9811428.

[19] Y. Chikashige, R.N. Mohapatra, and R.D. Peccei, Phys. Lett. 98B, 265 (1981).

[20] R. Shrock and M. Suzuki, Phys. Lett. 10B, 250 (1982). 
[21] A. Joshipura and S.D. Rindani, Phys. Rev. Lett. 69, 3269 (1992).

[22] A. Joshipura and J.W.F. Valle, Nucl. Phys. B397, 105 (1993).

[23] A. Datta and A. Raychaudhuri, Phys. Rev. D 57, 2940 (1998).

[24] T. Binoth and J.J. van der Bij, Z. Phys. C 75, 17 (1997).

[25] P.A. Baikov et al., in Proceedings of X Workshop on High Energy Physics and Quantum Field Theory (QFTHEP-95), Moscow, 1996, edited by B. Levtchenko and V. Savrin, hep-ph/9701412, p. 101; and E.E. Boos, M.N. Dubinin, V.A. Ilyin, A.E. Pukhov, and V.I. Savrin, 'CompHEP — Specialized package for automatic calculations of elementary particle decays and collisions,' hep-ph/9503280.

[26] H. Baer, F.E. Paige, S.D. Protopopescu, and X. Tata, “ISAJET 7.40: A Monte Carlo Event Generator for $p p, \bar{p} p$, and $e^{+} e^{-}$ Reactions,', hep-ph/9810440.
[27] CDF Collaboration, F. Abe et al., Phys. Rev. D 78, 4536 (1997).

[28] J. Ohnemus, Phys. Rev. D 44, 1403 (1991); U. Baur, T. Han, and J. Ohnemus, ibid. 53, 1098 (1996).

[29] S. Mrenna and C.-P. Yuan, Phys. Lett. B 416, 200 (1998).

[30] For statistics definitions used in this study, see Ref. [1].

[31] D. Hedin and V. Sirotenko, "Search for Higgs in $Z H$ $\rightarrow \nu \nu b \bar{b}$ channel in Run II: Analysis Study I,' DO Note No. 3519, 1998.

[32] R. Jesik, " $Z h \rightarrow \nu \nu b \bar{b}$ at the Tevatron Run II,' presented at the Tevatron Run II workshop on Higgs/Supersymmetry, 1998.

[33] W. Yao, "Search for $Z H \rightarrow\left(l^{+} l^{-}, \nu \bar{\nu}\right) b \bar{b}$ at CDF II," presented at the Tevatron Run II workshop on Higgs/ Supersymmetry, 1998. 